\title{
Accounting for tutorial teaching assistants' buy-in to reform instruction
}

\author{
Renee Michelle Goertzen, Rachel E. Scherr, and Andrew Elby \\ Department of Physics, University of Maryland, College Park, Maryland 20740, USA
}

(Received 29 April 2009; published 14 December 2009)

\begin{abstract}
Successful implementation of tutorials includes establishing norms for learning in the tutorial classroom. The teaching assistants (TAs) who lead each tutorial section are important arbiters of these norms. TAs who value (buy into) tutorials are more likely to convey their respect for the material and the tutorial process to the students, as well as learning more themselves. We present a case study of a TA who does not buy into certain aspects of the tutorials he teaches and demonstrate how his lack of buy-in affects specific classroom interactions. We would hope to design professional development programs to help TAs appreciate the power of tutorial instruction. However, our research suggests that the typical professional development activities offered to tutorial TAs are not likely to be effective. Instead, it appears that what we call the "social and environmental context" of the tutorials—including classroom, departmental, and institutional levels of implementation-has the potential to strongly affect TA buy-in to tutorials and probably outweighs the influence of any particular activity that we might prepare for them.
\end{abstract}

DOI: 10.1103/PhysRevSTPER.5.020109

PACS number(s): 01.40.Fk, 01.40.J-

\section{INTRODUCTION}

Experienced tutorial instructors and developers are well aware that successful implementation of tutorials includes establishing norms for learning in the tutorial classroom. These norms include an emphasis on conceptual understanding (and a concurrent de-emphasis of algorithmic application of formulas); an expectation that this understanding is best achieved through explaining one's own thinking, listening, and responding to others' ideas and constructing arguments; and an acceptance of instructors as facilitators of this process rather than sources of correct answers. The establishment of these norms is "among the most critical and subtle features of implementing these reforms." " From the students' point of view, the teaching assistants (TAs) who lead each tutorial section are important arbiters of these norms and expectations. The development of these norms by the TAs is thus a critical task of tutorial implementation. TAs who "buy into" tutorials are more likely to convey their respect for the material and the tutorial process to the students, as well as learning more themselves. This development is nontrivial: although TAs may be presumed to be more sophisticated learners than their students, they are in some cases more thoroughly embedded in traditional teaching practices.

We are conducting a project whose long-term goal is to design an effective professional development program for physics graduate students who are teaching tutorials. As we initially imagined it, such a professional development program would include activities and experiences to help the participants appreciate the power of tutorial instruction. We now suspect that typical professional development activities provided to TAs, such as completing the tutorial as if they were students and viewing pretest or posttest and Force Concept Inventory results, are not likely to accomplish this goal on their own. Our observations suggest that the social and environmental context of the tutorials-including classroom, departmental, and institutional levels of implementation and support-strongly affects whether TAs buy into tutorials and probably outweighs the influence of any particular activity or experience that we might prepare for them. We have chosen the term "social and environmental context" to emphasize two characteristics of the context: (i) the attributes affecting the particular situation come from both people and the environment and (ii) these characteristics are structural and have some permanence. We use the term "buy-in" to refer to the alignment of the TA's stated set of beliefs about how physics should be taught compared to the beliefs of the curriculum developers. Based on observations of tutorial implementations at the University of Maryland (UM) and University of Colorado-Boulder (CU), we argue that the social and environmental context at $\mathrm{CU}$ is more supportive of tutorials and tutorial instructors than the UM context. As a result, the TAs at $\mathrm{CU}$ buy in to tutorials more than the TAs at UM, which leads to specific identifiable consequences in the classroom.

In what follows, we first provide a detailed example of one way the lack of buy-in from a TA named Oscar undermines the effectiveness of tutorials. Next, we use the framework laid out by researchers at $\mathrm{CU}^{1,2}$ to consider how different levels of social and environmental context may affect the worth that TAs place on tutorials. Our observations highlight the need for further research on how professional development activities can support tutorial TAs in valuing reform instruction.

\section{RESEARCH ON TA INSTRUCTION AND TEACHER BELIEFS}

\section{A. Research on science TAs is limited and characterizes TAs' beliefs and teaching styles in general terms}

The physics education community has now produced many research-based undergraduate curricula that help students construct their own physics knowledge. ${ }^{3-6}$ The developers of these curricula have carefully studied how material should be presented and how students should best interact with it. Much less published research, by contrast, has focused on the TAs who, at many institutions, lead the discussion or recitation sections in which research-based curricula 
are implemented. For example, a classic pair of articles describing the development of two particularly well-studied tutorials ${ }^{7,8}$ describes the instructional environment in two paragraphs. The role of the instructors (who typically include TAs) is addressed as follows: "the instructors do not lecture but circulate throughout the room while the students work through experiments and exercises. A high instructor-tostudent ratio allows the staff to engage students in dialogues that permit in-depth questioning." 8

Much of the limited literature on science TAs has characterized TAs with only the broadest of descriptions. Research that presents detailed descriptions of the development and implementation of professional development programs $^{9-13}$ has often assessed the effect of TA participation in such programs by surveys or written assessments ${ }^{12,13}$ or with limited observations and/or interviews. ${ }^{9,11}$ Case studies afford more nuanced descriptions of individual TAs, but the cases rarely include detailed descriptions of classroom interactions to allow a fine-grained analysis of individual actions. ${ }^{14,15}$

\section{B. Research on teachers' beliefs has demonstrated their effect on implementation of reform curriculum}

While the literature on science TAs is very limited, the large body of research on teachers and their beliefs is a useful place to begin identifying influences on teachers' practice in the classroom. Numerous studies have shown that instructors' beliefs about their abilities as teachers, about how their students learn, and about whether they are in a supportive environment affect how reform curricula and methods are used. Case studies of math and science teachers provide examples of teachers who modified provided reform curricula to better fit their beliefs about how their students best learn. ${ }^{16-19}$ Similar modifications were made by teachers on the basis of their beliefs about their own abilities and the support (or lack thereof) from their school environment. ${ }^{20}$ Likewise, instructors' beliefs about the nature and purpose of formative assessment were seen to influence how it was used in the classroom..$^{21}$ (For a fuller description of teacher beliefs and their influence on teaching, see Refs. ${ }^{22,23}$.)

\section{Research has shown that reformed teaching correlates with student learning}

The ultimate goal of TA professional development is increased student learning. Research has demonstrated that reformed teaching, as measured by the Reformed Teaching Observation Protocol, correlates significantly with improved performance on the Lawson Classroom Test of Scientific Reasoning. ${ }^{24,25}$ Other studies have found that student gains are positively correlated with instructor participation in professional development designed to encourage a particular kind of "constructivist" teaching ${ }^{26,27}$ and with instructor use of constructivist teaching. ${ }^{28}$ An extensive literature review by Close $^{29}$ found that reformed teaching is the only teacher characteristic that is reliably correlated with student learning; studies of other positive teacher characteristics, such as more sophisticated nature of science beliefs or more years of schooling, have had mixed or inconclusive results.

\section{THEORETICAL FRAMEWORK}

\section{A. Epistemological framing}

Framing is a construct developed in anthropology and linguistics ${ }^{30}$ to describe how an individual or group forms a sense of "what is it that's going on here?"31-34 To frame an event, utterance, or situation in a particular way is to interpret it based on previous experience: to bring to bear a structure of expectations about a situation regarding what could happen, what portions of the information available to the senses require attention, and what might be appropriate action. For example, monkeys engaged in biting each other are skilled at quickly and tacitly "deciding" whether the biting is aggression or play. An employee may frame a gift from her supervisor as kind attention or as unwelcome charity. A teacher may frame a physics problem as an opportunity for sense making or as an occasion for rote use of formulas. In school settings, epistemological framing is of particular importance: students and teachers form a sense of what is taking place with respect to knowledge, including, for example, what portions of information and experience are relevant for completing assignments. Other aspects of framing are important as well, including social framing, in which teachers and students form a sense of what to expect of each other and of themselves during interactions. For individuals working together collaboratively, the social and epistemological aspects of framing interact.

A frame becomes stable when the activated network of cognitive resources (elements of thought) are reinforced by each other and/or by social and material cues. We argue at the end of Sec. VI that some of Oscar's behaviors as a tutorial TA reflect an epistemological frame whose stability arises from feedback loops among the underlying "beliefs," attentional focus, and patterns of action. This explanation differs from an account wherein behavioral patterns result from the global robustness of the teacher's epistemological (and other) beliefs. ${ }^{16,20,35}$ By our account, we also expectand, in fact, observe- a fair degree of context dependence within a given TA's cognition and classroom behaviors at various grain sizes. Some aspects of Oscar's teaching, not discussed in the paper, suggest this kind of variability. More importantly for us, in Sec. VIII, we argue that specific social and material cues-specific components of the social and environmental context-affect how TAs frame their tutorial teaching.

In this way, framing is a useful construct for bringing both local action and more indirect contextual influences into explanations of TAs' behaviors. To the extent that framing is an interpretation based on previous experience, it is informed by an individual's broad history and experience with related events and systems. In the moment, though, participants mutually construct their sense of shared activity by means of verbal and nonverbal interactions, including linguistic signals, prosodic features, and body language. ${ }^{31}$ Participants' understanding of the nature of the activity in which they are engaged-i.e., their framing of the activity - guides their selective attention, provides cognitive structure for interpreting events, and manifests itself in their observable behavior. ${ }^{36}$ 


\section{B. Explanatory trade-offs between direct local and indirect contextual factors}

Our use of framing is informed by Erickson, ${ }^{37}$ who emphasizes a trade-off between scope and specificity in explanation of behavior. Empirical study of specific interaction has crucial advantages: rich data, depth of analysis, and the hope of accounting for moment-to-moment actions, as we attempt in Sec. VI. This depth and explanatory power, however, comes at the expense of scope. In order to study how TA behavior unfolds in real classroom situations, we must choose a particular TA in a particular classroom at a particular time, interacting with a particular set of students. Studying specific episodes does not allow us to predict how other TAs would behave in similar situations or even how our focal TA would behave in different situations.

By contrast, in our Sec. VIII analysis of social and environmental influences on TAs' behavior, we emphasize scope while sacrificing specificity. We can argue that social forces affect TAs in particular ways, but heeding Erickson, we do not try to show how that large-scale effect plays out in local situations. For example, the "upstream" influences that shaped a TA's behavior-what may have taken place in his history to cause him to behave as he does in the present-are not visible on videotape of his classroom behavior. Neither are the pressures he may be feeling from entities outside the classroom.

\section{INSTRUCTIONAL CONTEXTS}

\section{A. University of Maryland}

\section{Course description}

The TAs described in this study taught tutorials which took place as part of a two-semester algebra-based introductory physics course at the University of Maryland, with approximately 160 students in each lecture section, most of whom are junior and senior health and life science majors. The students, over half of whom are female, reflected the wide ethnic diversity of the University of Maryland. Lectures are held two or three times a week in a large lecture hall and approximately 100-200 students comprise a lecture section.

The course was reformed as part of a project titled Learning How to Learn Science: Physics for Bioscience Majors, carried out at the University of Maryland from 2000 to 2005. The project adopted reforms that were well documented to produce conceptual gains and adapted them to try to create a coherent package that also produced epistemological and metacognitive gains. ${ }^{38}$ However, most of the lecture sections (four out of the six) were not taught by physics education research (PER)-affiliated professors and thus the lecture instruction was largely traditional in those sections.

As part of the course reform, the traditional teachingassistant-led recitation was replaced with worksheet-based group-learning activities ("tutorials") based on the model developed at the University of Washington. ${ }^{5,39}$ In the tutorial sessions, students worked in small groups on worksheets that led them to make predictions and compare various lines of reasoning in order to build an understanding of basic concepts. TAs served as facilitators rather than as lecturers. Each class section consisted of six groups of four students each, supervised by two TAs. The tutorials were constructed to emphasize the reconciliation of everyday intuitive thinking and experience with formal scientific thinking, as well as to encourage explicit epistemological discussions about the learning process. ${ }^{40,41}$

\section{Teaching assistants}

The majority of the UM tutorial TAs who participated in this study are first or second year graduate students assigned to work as teaching assistants to support themselves before joining a research group. Most are chosen as a matter of convenience, not because they or the lecturer requested the assignment. A few are upper level graduate students who had unfunded research positions. Most are in their early twenties. During the two semesters of this study, the only women assigned to teach tutorials were physics education graduate students, who were excluded from the study. Thus, all the TAs in the study are male. They live in a suburban metropolitan area and attend a competitive research university with a large undergraduate and graduate physics program. Although almost half of the TAs who participated in the study are not native speakers of English, all but one communicate easily in English. Most of the TAs had entered graduate school immediately after their undergraduate studies, and only one (who had taught high school) had experience teaching beyond tutoring or leading discussion sections.

\section{Tutorial preparation sessions}

The UM tutorial preparation sessions are weekly one hour meetings in which TAs prepare to teach the next week's tutorial. The TAs sit in groups of two to four at tables in the tutorial room. The session usually begins with a discussion of content problems the students had during the previous week or with a conversation about classroom management issues, which lasts from ten to thirty minutes. The TAs spend the remaining time working on the upcoming tutorial. The tutorial supervisor circulates, modeling appropriate instruction techniques and highlighting anticipated student difficulties.

\section{B. University of Colorado}

\section{Course description}

The teaching assistants who participated in this study taught tutorials associated with a two-semester calculusbased introductory course at the University of Colorado, Boulder. The students in these courses are mainly engineers and natural science majors. More than half of the students are freshman, and $75 \%$ are male. Lectures are held three times a week in a large lecture hall and approximately 200300 students comprise a lecture section. This course was reformed during a large-scale project from 2003 to 2007. These reforms included increased use of research-based methods such as concept tests in lecture and small-group activities. They also focused on sustaining these reforms across multiple lecture instructors, including those not asso- 
ciated with the physics education research group. The result has been increased conceptual gains. ${ }^{1,2}$

A significant part of this reform was the implementation of tutorials, the worksheet-based group activities developed by the University of Washington, ${ }^{5,39}$ which replaced traditional discussion sections. Students work in small groups to complete ungraded worksheets that lead them to build their own understanding of basic physics concepts using discussion and prediction. The students complete a pretest to elicit preconceptions before their tutorial section and are assigned tutorial homework after the section. At CU, each tutorial section is taught by one TA, a graduate physics student, and one learning assistant (LA), an undergraduate student who attends a semester-long course on theories of learning and teaching methods. Each tutorial section has six or seven groups of four students each.

\section{Teaching assistants}

The CU TAs who participated in this study were all firstyear graduate students who had taught tutorials in the fall (either in mechanics or electromagnetism) and were assigned to teach the tutorials for the introductory mechanics course during their second semester. Some had previously tutored, but none had taught in a classroom before the current year. The TAs who participated were all male and in their early twenties. One was a non-native speaker of English but communicated easily in English. The TAs live in a suburban area and attend a competitive research university with large graduate and undergraduate physics programs.

\section{Tutorial preparation sessions}

The CU tutorial preparation session takes place two days before the tutorial is taught and is attended by both TAs and LAs. The TAs are expected to arrive fifteen minutes earlier than the LAs, so that they can discuss the grading of the upcoming tutorial homework with the tutorial supervisor. After that, the TAs and LAs review a tutorial pretest that the students have completed. The remaining time, between thirty and forty minutes, is spent working through the upcoming tutorial. The tutorial supervisor circulates, sometimes discussing administrative issues and sometimes discussing the content of the tutorial. Unlike the situation at UM, the lecturer associated with the course often attends portions of the preparation sessions, modeling instruction techniques and answering questions.

\section{DATA COLLECTION AND SELECTION OF EPISODES}

\section{A. Data collection at the University of Maryland}

\section{Classroom video}

At the University of Maryland, tutorials are held in a single room with six tables at which students work collaboratively in groups of four. Students typically do not move their seats during the class session or even from week to week. We try to keep the recording of the tutorial activities subordinate to normal classroom practices, so two small Hi-8 or mini-DV video cameras on tripods are positioned on the periphery of the room, each focused on a single table. The cameras do not move. Microphones are embedded in cages on the tables that are being recorded. A researcher turns on the cameras at the start of the tutorial session, but the cameras are otherwise unattended. Our intention is to make the video recording as unobtrusive as possible, even at the expense of visual or sound quality.

\section{Interviews}

At the beginning and end of the first semester, TAs were interviewed by a PER researcher who was not associated with the TA training at UM. The interviews were either audiotaped or videotaped and lasted approximately one hour. A list of approximately ten open-ended questions was used as the starting point for the interview, which included questions about the TA's past teaching experience, advantages and disadvantages of tutorials compared to traditional discussion sections, and suggestions about how to make tutorials better. (Appendix B lists all the questions.) If TAs wished to discuss other topics relating to tutorials or the course we pursued those conversations first. In general, all the topics in the protocol were covered during the interview, either in response to the interviewer's questions or during discussions of issues raised by the TA. In our coding of TA buy-in, we used all portions of the interviews in which TAs discussed their opinions of tutorials; discussions of the TAs' teaching histories, their comments about the lecture or laboratories, or their evaluations of how the students viewed tutorials were not included. However, these comments were included in our case study of Oscar.

The purpose of interviewing TAs at the beginning and end of the first semester was to have the opportunity to observe changes in their values. We observed, however, that some TAs' values changed a little and some showed no change (as detailed in Appendix A). For this reason, we combined the data from the initial and final interviews. The observation that UM TAs' values did not change during their first semester of teaching challenges the model implicit in UM's TA preparation program because we expected that the TAs' classroom experiences would be a primary influence on their beliefs. That is, it was hoped that as TAs participate in reform instruction, they would begin to value it. ${ }^{42}$ Our data, in contrast, are more consistent with an account in which a given context (such as a tutorial classroom) evokes beliefs and actions that are stable and not easily modified.

\section{B. Data collection at the University of Colorado}

\section{Classroom video}

The CU tutorials are conducted in an enormous room that contains areas for laboratories, homework assistance, discussion sections, and three subdivided bays for the tutorials. The tutorials for each course are all held on the same day of the week, so generally there are two or three occurring simultaneously. Each bay has seven or eight tables at which groups of four students work collaboratively. Like the taping at UM, the CU taping was arranged to minimize disruption to the students. Two tables were taped in each participating section by a mini-DV camera that was placed at least twelve feet 
away. There were small microphones taped to the middle of the table. Because tutorials occurred in different rooms, the microphones had to be taped down and cameras turned on in the few minutes before each class. The researcher made an effort to do this when only a few students were in the classroom to avoid disturbing students and to make the recording as unobtrusive as possible. In most cases, the same tables were taped each week, but occasionally a different table was taped because it was more convenient.

\section{Interviews}

Because data collection at $\mathrm{CU}$ took place for only one month toward the end of the semester-long course, the TAs participated in one interview (in contrast to two interviews for UM TAs). The interviews were videotaped and took about one hour. The protocol for the CU TAs was the same as that used for the UM TAs.

\section{Selection of TAs}

At UM, 17 graduate students over two semesters agreed to be taped during their tutorial teaching. At $\mathrm{CU}$, four graduate students and two undergraduate student TAs (who were participants in CU's learning assistant program) agreed to be taped while teaching. A total of 15 UM TAs, 4 CU TAs, and 2 CU LAs agreed to additional participation in the project, which included the completion of two interviews and one survey. They received a small stipend for this additional participation. We did not study TAs who were affiliated with either PER group, although some TAs chose to work with the PER group as research assistants after their participation in this project was completed.

As part of our larger project we chose a smaller subset of TAs to study in more detail. These TAs were purposefully chosen because they seemed articulate about their teaching or their students in preparation meetings or in their interviews. We excluded LAs at $\mathrm{CU}$ because we viewed their experience as undergraduates with additional training as sufficiently different than the graduate TA population that was the focus of this study. We watched multiple clips of each TA interacting with students on video, seeking to describe and generate plausible explanations for the TA's action.

\section{Selection of video episodes}

During the two years we collected data, we videotaped 19 sections of introductory courses at UM, covering the entire semester of the introductory course. This resulted in approximately 340 hours of video. At CU, 18 sections were taped during one month of observations, which produced approximately 70 hours of video. The two episodes that are described in detail in this paper were chosen while we were viewing numerous clips from a single TA, a process that we have repeatedly used to more deeply understand the classroom practice of individual TAs. These clips were chosen because they clearly illustrated the ways in which a TA's beliefs about tutorials influenced his use of them.

\section{EXAMPLE OF TA BUY-IN AND ITS EFFECT ON CLASSROOM INTERACTIONS}

Effective tutorial teaching requires TAs to support a variety of pedagogical ideas. For example, instructors need to maintain an environment in which students work in groups and TAs do not give students solutions. TAs must also value conceptual understanding and should encourage students to construct their own knowledge. In practice, we would expect that different TAs would buy into these different components of tutorial instruction to varying degrees. Thus their valuation of tutorials would not be along a simple continuum but would require a more complex characterization.

In this section, we present a case study of a TA who buys into some but not all of the components of the tutorials. Our analysis aims to establish two points:

(i) A TA's lack of buy-in directly affects his instruction, including his in-the-moment interactions with students.

(ii) This instructional effect can stem from comparatively subtle fine-grained lack of alignment with the developers' intentions. The lack of buy-in need not be a blanket rejection of the entire tutorial approach to instruction.

Oscar is a UM physics graduate student who taught the introductory physics course for three semesters during his first two years of graduate school. He feels a sense of responsibility toward his job and wants his students to succeed. He was initially assigned to the introductory course, but he chose to teach tutorial-based courses two more times. In his interview, he explains that his initial experience teaching tutorials better prepared him to ask students questions that could help them and that he considers the class more fun than doing problems at the board, as he would have done in a typical recitation section. In addition, he expresses concern that students did not learn as much as they could from the class because they did not pick up their graded homework from him. So, although Oscar does not buy into many components of the tutorials, this is not due to a dislike of his job or a lack of concern about his students and their learning.

\section{A. Oscar's expression of his tutorial values in interviews}

Oscar values some parts of tutorials: he considers group work, conceptual understanding, and the role of the TA as a questioner to be important aids to student learning. However, he does not buy into some aspects of the tutorials that the developers consider essential, including the value of starting with and refining everyday thinking when learning formal physics concepts and of TAs continuing to learn both physics and instructional methods. (An indication of the amount of Oscar's buy-in is found in Appendix A in the column labeled "O.") Oscar's lack of buy-in is in some ways subtle: a cursory inspection of his teaching practice would show a TA who sometimes questions his students as they work in small groups and who at other times patiently waits at a side table while students work through the tutorials on their own. But, as we will demonstrate in Sec. VI B, the tone he establishes and the message he sends to his students with respect to the tutorials are profoundly different than what the developers intend. 


\section{Value some basic premises of tutorial learning}

a. Group work. In his interview, Oscar says, "Group work, I think, is very beneficial in that people are actively thinking about it. If they're working on a homework problem together, they can, you know, they can curb each other's stumbling blocks and explain it to each other. And, you know, once you've explained something to somebody, it's a lot easier to understand it yourself." Oscar believes that group work helps students with difficulties because their classmates can assist them and because articulating ideas can help you learn them.

For Oscar, having a TA available is an important part of the group work. He says that the tutorial setup lets "...them work together, there, with a TA present, so that if they do as a group have a stumbling block, there's someone who can, who can get them all through it together. And then, probably just for enough so they can keep working on it on their own." Thus, Oscar expects that students will encounter problems that can only be solved with the TA's assistance; but the assistance should be minimal, "just enough" to get students unstuck. He also notes that he values group work in his own studies. He explains, "We're always working together on the homework.... And it works, it works wonderfully." The various reasons Oscar provides show that his level of buy-in to group work is quite deep: he perceives its value for students of any skill level, and he has experienced the benefits himself.

b. Comparison to traditional discussion sections. Oscar sees the tutorials as more beneficial to students than traditional problem-solving discussion sections. He said that such a discussion section would be a "boring problem for me, and they probably still don't get it." He saw tutorials as a way to elicit specific problems his students were having. He summarized his feelings by saying, "So overall, I think it's a good approach, it's a good method, and I bet it's effective."

\section{Is ambivalent about some features of tutorial learning}

a. Conceptual along with quantitative. Oscar values both conceptual and quantitative understandings of physics, but he thinks that because tutorials focus on conceptual learning, the students do not get practice integrating the two types of knowledge. He says, "I mean, every physics intuition I have, I'm almost certain I've gained by having a very vague idea at first, doing some problems, and then seeing how it relates. Going back to the idea, thinking about it a little more, in light of the results of these problems..." This quote also expresses Oscar's idea that solving quantitative problems is a way to create qualitative understanding. As he says, "I think if they just had the formula given to them... even if they don't understand it. Give them the formula. Give them some numbers to plug in. And they might see, oh, it doesn't really matter if there's this thing above it or not. And, I think if they got that kernel of wisdom, then they might start to think, oh, why is that?" Many physicists build up their qualitative understanding of the subject with minimal scaffolding while doing problem sets, so it may be that Oscar thinks that the best way for students to develop their conceptual understand- ing is by first working out quantitative problems and then reflecting on the results, as physicists do. Thus, Oscar agrees that students need to develop a conceptual understanding of physics, and he sees it as a job they need to do themselves. Where he disagrees with the tutorials is the method by which students can best do this; Sec. VI A 3 a will more fully discuss this.

b. TA as questioner and coach. Oscar also expresses mixed feelings about the value of TA questions in student learning. In his interview, he said that it was important for students to work through problems themselves rather than have the answer given to them. He felt that his questions could help the students if they provided a broader context for the problem the students were working on. However, he conveyed frustration with the use of questions in some instances. For example, in the TA training the TAs were told to paraphrase questions back to students. The purpose of this instruction was to provide TAs with a way of checking their understanding of the student's question, but Oscar said that graduate students were sufficiently prepared to comprehend students' questions and that this would only assist a student who "is so lazy that they really need to hear their own question repeated back to them to get them to think about it." At another point, Oscar also communicates disappointment that he is prevented from giving students answers directly when he says, "Sometimes... they really just need to be given the answer. If they're just given the answer, you know, they know what to work towards, and maybe that's the bigger picture they need." Oscar's view here is nuanced: he does not think that giving students the answer will make everything clear. Instead, he wants to give students answers because he thinks that they can be a foundation upon which students can build their own understanding. By contrast, the curriculum developers, who included Scherr and Elby, were afraid that many students would simply accept the provided answer and move on rather than building from it as Oscar intends. Although observations of Oscar's teaching show that he often questioned students, the interview suggests that he felt constrained by this method.

\section{Does not value certain aspects of tutorial learning}

As the past sections demonstrated, Oscar buys into tutorials at the coarse-grained level typically emphasized in professional development training offered to TAs. He agrees that small-group interactions, assisted by TAs, are an effective method for teaching, and he believes that TA questioning is sometimes a useful tool for helping students. However, there are aspects of the tutorials that Oscar does not value as much: the strategy of building from common-sense ideas toward formal physics understanding, the need for TAs to continually learn more about the physics they teach, and the level of challenge appropriate for introductory students.

a. "Fake" concepts. As detailed in other publications, ${ }^{38,40,43}$ UM tutorials emphasize students' epistemological development by focusing on how their knowledge from everyday life and other subjects can connect to what they are learning in their physics course. In particular, tutorials en- 
courage students to start with their everyday thinking and then refine that thinking toward a correct understanding of the targeted physics concept. Consequently, some tutorials may introduce concepts using noncanonical terms or encourage students to discuss whether they expect physics to make sense all the time or if equations should match their common sense. This aspect of UM tutorials is particularly jarring for many TAs, including Oscar.

Oscar expresses concern that asking students to use everyday experiences as a basis for building physics knowledge is not productive. Instead, he thinks that students more effectively learn when they are exposed to the scientifically accepted knowledge, which they must then make sense of and check against their everyday ideas. He cites a particular example in the sixth tutorial of the semester, which introduces the intuitive idea of "oomph." The students are told, "The more oomph something has, the harder it is to stop, and the more ability it has to knock things over." They are led through a series of questions that help them construct a formula for oomph and are then told that oomph corresponds to momentum. Oscar proposes a different approach: "If they can all at least agree that oomph is something that's going to be called momentum, that's going to be something $m v$, I think that would kind of cement their thinking together." $\mathrm{He}$ adds, "But something like oomph, which is, ah, it's fake, it's nothing real. It's not even something people have a real concept of. And most of them have studied physics already." In this case, Oscar does not think that students' experiences from everyday life will help them understand the idea of momentum as it is used in the course. His comment suggests that he does not see value in using a noncanonical term and that he assumes students can find more meaning when given an equation as compared to when they generate the equation themselves, at least in this case.

b. TA as learner of physics. A TA could view the weekly tutorial preparation meetings as providing an opportunity to learn physics in a deeper way, as one CU TA mentioned he does. TAs who feel that doing tutorials helps them learn physics in addition to learning instructional techniques are in a better position to appreciate tutorials as they appear to the students. While Oscar does not discuss the preparation meetings during his interviews, his participation in these meetings indicates that he does not view them as a productive activity. In general, Oscar participates in the discussions about students, demonstrating that he seems to consider it appropriate to discuss difficulties regarding classroom management or particular content problems students have.

However, during the portion of the meeting where TAs complete the tutorial itself, Oscar does not regularly participate in the discussion about the correct answers to the questions or in discussions when TAs anticipate student answers, although he sometimes makes humorous off-the-cuff remarks. In one preparation meeting, for example, Oscar is working with two other TAs on a tutorial about torque. Oscar participates minimally in the discussion: he answers the questions on the worksheet in a monotone and sits slouched at the table with a neutral expression. His most animated contributions are jokes and critiques of how the questions are worded. At one point, Oscar takes the paper clips that the tutorial intends to be hung on a balance and forms them into a chain; he attaches a washer to the end of the chain and swings the assembly rhythmically back and forth. His activity makes the paper clips unavailable for their intended purpose, as another TA complains, "We need more paperclips. Stop being such a paperclip hog." Overall, Oscar's posture, tone, and activities communicate his feeling that the TA meeting will not be a useful experience for him.

c. Correct level of challenge for introductory students. Oscar is concerned that what the tutorials ask students to do is too difficult for them. He says, "So I think doing that continuously, you know, making the students expect to have to just trudge through all this stuff, that they, you know, that they don't really understand and not really sure where they're going, week after week is, is overtaxing on them." Later, he contrasts the tutorials to repeated quantitative problem solving: "It's a mistake to try and force them into this new way of doing physics right away... They need to be eased in. You know, give them, give them ten plug and chug problems first. Just so they can get used to doing some of the math and some of the concepts." Oscar is not buying into the tutorials' assumption that introductory students, some of whom are accustomed to problem solving by rote, can adapt to a style of learning which requires them to be active participants.

As shown in the earlier section on group work, Oscar buys into the idea that students need to construct their own physics knowledge. He thinks that students teaching themselves is "really the only way to really learn something." However, he believes that students need different amounts of assistance from a TA in order to build that understanding. He explains, "For particularly good students, I think that they have the potential from the beginning to just think through things, and giving them the answer might satisfy them temporarily, but not really get them thinking... But I think for students maybe in the middle, sometimes just being able to tell them, 'Listen, this is how it works...' I think in some cases that would help." While Oscar believes that successful learning only takes place when students do the work themselves, he does not think that the average student can do that work without help from the TA. He believes that "help" can include providing the answer and that "thinking through things" can include making sense of that answer.

These examples demonstrate that Oscar cannot be simply classified as buying in or not buying in to tutorials because his support for the tutorials depends on the particular attribute being considered. In particular, he views the tutorials' focus on conceptual understanding as important and he values group work in part because it fits with his epistemological ideas that students must construct their own knowledge. However, he disagrees with the particular epistemology enacted by the UM tutorials: that learning physics is the refinement of everyday ideas and should therefore start with everyday ideas. It is likely that tutorials based on more formal concepts would get more buy-in from Oscar. 
Before accepting that there's an irreconcilable contradiction between Newton's third law and the intuition that the car reacts more during the collision, let's try a reconciliation stategy called refining your intuitions.

We'll start with a new question. Suppose the truck's mass is $2000 \mathrm{~kg}$ while the car's mass is $1000 \mathrm{~kg}$, and suppose the truck slows down by $5 \mathrm{~m} / \mathrm{s}$ during the collision. Intuitively, how much speed does the car gain during the collision? (Apply the intuition that the car reacts more during the collision, keeping in mind that the truck is twice as heavy.) Explain your intuitive reasoning.

FIG. 1. Excerpt of the UM tutorial on Newton's third law.

\section{B. Oscar's expression of his tutorial values in the classroom}

Sections VI B 1 and VI B 2 will examine samples of Oscar's classroom interactions and how his buy-in (or lack of buy-in) of specific tutorial attributes influences his instructional behavior. The observed interactions occurred in the tutorial classroom during the semester Oscar was teaching tutorials for the third time.

\section{Newton's third law tutorial}

In this episode, Oscar's class was working though a tutorial that helps students reconcile the idea that two colliding objects each feel the same force (Newton's third law) with the "common-sense" idea that a larger truck causes more damage to a smaller car when they collide than vice versa. ${ }^{40,41}$ The tutorial begins by considering the collision of a truck and a stationary car. The students were asked to use their common sense to generate a guess about which vehicle experiences a greater force. After the students read an explanation of Newton's third law, they applied the law to the situation and then observed two carts colliding, with force probes attached, as a demonstration of Newton's third law. The tutorial then poses the question excerpted in Fig. 1. A correct answer would be that the car gains $10 \mathrm{~m} / \mathrm{s}$ because it weighs half as much as the truck and so it will react twice as much.

a. Instructs all students to disregard the term common sense. Oscar communicates his lack of buy-in to the tutorials with instructions he gives to his class. At the start of this tutorial hour, Oscar instructs the whole class: "In part one, it says 'common sense,' feel free to replace that with 'a guess.' Um, when I, when I went through this I thought this isn't common sense at all." This instruction conflicts with the intention of the tutorial because the tutorial deems the common-sense idea that the smaller car will be more damaged as a reasonable idea that students will likely hold and requires that this idea not be discarded but rather reconciled with Newton's third law.

Oscar's exhortation here aligns with his beliefs regarding the usefulness of intuition when learning physics. His correction is aimed at the tutorial's intention to encourage students to refine and build on their common-sense ideas. In his interview, Oscar expresses his belief that everyday ideas are simply different than physics ideas and that the time to reconcile (to the extent possible) is after the student has learned and practiced using the formal concepts. When he tells his class to "guess," he may be suggesting to them that their task is to fill in a space on a worksheet, not generate an idea on which they can productively build.

b. Declines the opportunity to support particular students' common-sense reasoning. In another episode, a group of four students has called Oscar over and told him that they think the answer to the exercise above is $10 \mathrm{~m} / \mathrm{s}$. Oscar affirms this and then asks them "Why?" which leads to the following conversation. The students offer their reason that "the car is half," which Oscar interprets as saying the car has half the mass of the truck. He prompts them to think about quantities that are the same and different before and after the collision. The students then answer that the force is the same and the things that differ are velocity and acceleration, which Oscar then connects to the equation $F=m a$.

$1 \mathrm{~S} 4$ : The mass, the mass that describes it...

2 Oscar: They're-

3 S4: One is, the car is half. Yeah.

4 S2: One is twice as much.

5 Oscar: Right. Okay, so they're different

6 masses.

7 S1: Right.

8 Oscar: Meaning...

9 S2: They're going to have different... um...

10 Oscar: I mean, you're on the right track.

11 S2: Do you want us to talk about inertia?

12 Or...

13 Oscar: Uh, inertia is a little, just a little bit

14 beyond this. I mean, if you can think of it

15 in terms of inertia, that's fine. But that kind

16 of, uh, makes answering these questions

17 harder. The basic problem in pretty much

18 every last physics question you'll ever

19 answer is to figure out what's the same and

20 what's different. Either before or after. So

21 in this situation, what's the same?

22 S2: The force.

23 Oscar: Okay, and what's different?

24 S2: The mass.

25 Oscar: Is that it?

26 S4: The velocity.

27 Oscar: And the-is that it?

28 S1: Yes.

29 S2: I don't know.

30 S1: No.

31 Oscar: No. You're on the right track. You're

32 close. 
$33 \mathrm{~S} 2$ : I feel like this question is designed to

34 make us feel stupid.

35 Oscar: Ah, yeah. They are, they are. That's

36 why I told you guys, don't worry about

37 calling it common sense. It's a guess.

38 S1: Right.

39 Oscar: Right?

40 S2: Um...

41 S4: Friction?

42 Oscar: Say what?

43 S4: Friction?

44 Oscar: The what?

45 S4: Friction.

46 Oscar: Oh, no, no, don't worry about

47 friction here.

48 S2: The acceleration, maybe?

49 Oscar: Yeah. Right. So what is, do you

50 guys have a formula for the force?

51 S3: Ah, mass times acceleration.

52 S4: Acceleration.

53 Oscar: Yeah.

Oscar guides the students to the reasoning that he considers appropriate rather than the reasoning that the tutorial is trying to elicit. After the interaction discussed here, he eventually prompts the students to say that because the forces are the same and because force is equal to mass times acceleration, the fact that the mass of one of the objects is greater means that its acceleration must be less. Here Oscar is using the idea that the forces are equal and the relation that force is equal to the product of mass and acceleration to show that in a collision, one object could have a greater acceleration than the other if its mass is smaller.

The instructional moves Oscar makes in this episode align with his epistemological belief that everyday ideas are not a useful foundation for building physics knowledge. The students' answer of $10 \mathrm{~m} / \mathrm{s}$, supported by their common-sense idea that the car will speed up twice as much as the truck slows, because the car is half as massive, is a response that the tutorial developers consider appropriate. The students' ideas about inertia or the difference in the vehicles' masses, if elaborated, would also be approaches that could feed productively into the subsequent exercises in the tutorial. Oscar, however, rejects the students' ideas as insufficient because he does not buy into the idea of intuition refinement. Instead, he expects a compensation argument between mass and acceleration with respect to the equation $F=m a$. The tutorial also (eventually) wants students to make this argument, but it wants this to connect to their common-sense idea that the car speeds up twice as much as the truck slows down, a connection that Oscar does not value.

Oscar's intervention here also aligns with his beliefs that physics knowledge needs to be constructed by making sense of equations. Our observations of his teaching in other tutorials show that he does not often alter tutorials in a broad way, as he does by announcing this change to the entire class. It seems unlikely that he has made this change simply because he enjoys modifying tutorials or because he is responding to a specific student's need. Instead, the change that he has made aligns with his beliefs about how physics should be taught.

c. Positions himself away from the tutorial developers. In the encounter described above Oscar positions himself as separate from the tutorial developers, who designed the questions that make the students "feel stupid." By agreeing with Student 2's assessment, Oscar indicates that he does not think that what the students are being asked to do will help them. He is not introducing this attitude to the group; before Oscar approached the group, Student 2 had remarked, "They're assuming that we're a lot dumber than we really are," and other students made comments that supported this sentiment. Even so, Oscar reinforces the students' discomfort by implicitly telling them, through his questioning, that they are not approaching the problem the right way.

\section{Oomph tutorial}

We analyze another exchange that occurs when Oscar's class is working through the sixth tutorial of the semester, which introduces the concept of momentum. As discussed in the section examining Oscar's valuation of tutorials, this tutorial introduces the intuitive idea of oomph and then asks students to develop a formula to represent its dependence on mass and velocity. It then connects that concept and formula to the formal idea of momentum. Example collisions give students the opportunity to show that the equation matches their intuitive ideas.

In the episode below, the students have completed the first third of the tutorial and reached a point where they are supposed to consult with their TA. They wait about ten minutes, at which point Oscar notices that they are not working and approaches them. He asks what momentum is, and the students respond that it is $m v$. He asks for more reasoning, and they explain why they would expect momentum to depend on mass and velocity. Oscar acknowledges this and discusses why it makes sense that the two quantities are multiplied (instead of divided, for example). He then draws their attention to the fact the momentum is a vector quantity. In the second half of the episode Oscar introduces examples to focus on why momentum depends linearly (as opposed to quadractically, etc.) on mass and velocity.

1 Oscar: How are you guys coming along?

2 Are you about at a checkpoint?

3 S1: I think so.

4 S?: Yeah. [2 s pause] 
5 Oscar: All right, so what's, uh, what's

6 momentum?

$7 \mathrm{~S} 1: p$.

8 Oscar: Okay, $p$ equals...

$9 \mathrm{~S} 2: m v$.

$10 \mathrm{~S} 1: m v$.

11 Oscar: Okay. And why $m v$ ?

$12 \mathrm{~S} 1$ : Because it depends on the mass and

13 velocity.

14 Oscar: Okay. That's a good start. So

15 some function of mass and velocity. Why

16 is it, why is it $m$ times $v$ ?

17 S1: Um...

18 S4: Cause you're measuring how, how

19 the, how something is moving-

20 Oscar: Okay.

21 S4: - toward the same direction. So the

22 components would be the mass of the

23 thing that's going and how it's moving,

24 which is the velocity. [3 s pause]

25 Oscar: Right, but why're they in that, the

26 question is why are they in that particular

27 relationship. What happens if you, if you

28 just change the mass, but leave velocity

29 the same?

30 S2: The momentum will change?

31 Oscar: Right. Right, let's say you double

32 the mass, how's the momentum change?

33 Students: Doubles.

34 Oscar: Right. You double the velocity,

35 and...

36 S3: Double.

37 Oscar: Yeah. Doubles. As long as you

38 keep the mass the same. Right. So, so,

39 they're somehow on equal footing, right?

40 So I mean if you divided one by the

41 other, that wouldn't really make as much

42 sense. If you subtracted one from the

43 other, I mean, I didn't mean [inaudible] it

44 just wouldn't make any sense.
45 S1: Yeah.

46 Oscar: So you think of it as $m v$. Um,

47 what kind of number is $p$ ?

48 S4: Um...

49 Oscar: Scalar, vector, tensor, bilinear?

50 S4: Vector.

51 S1: Vector.

52 S4: Vector.

53 Oscar: Vector? Why is it a vector?

54 S1: Cause it has direction.

55 S4: Direction.

56 Oscar: Well, a lot of things can have

57 direction. And they don't have to be

58 vectors.

59 S1: Um, and a magnitude?

60 Oscar: Say what?

61 S1: And a magnitude?

62 Oscar: Yeah, exactly. Yes, it's pointed

63 and it has magnitude. Well, it's, it's

64 important. I mean, you can, you can

65 point-

66 S1: Yeah, right.

67 Oscar: -in some direction, like an angle.

68 Like an angle is pointed in some

69 direction, but it's not a vector, right. All

70 right, so, um, the example I've been

71 talking with everybody else about is, um,

72 the bowling balls, if you have two

73 identical bowling balls and you, and you

74 push them off with the same velocity.

75 Each one has momentum $p$, right. How

76 much is $p$ ?

$77 \mathrm{~S} 1$ : How much is $p$ ?

78 Oscar: Yeah.

79 S1: It depends on their masses.

80 Oscar: Okay, let's say it has mass $m$ and

81 velocity $v$. How much is, uh, the

82 momentum of one bowling ball?

$83 \mathrm{~S} 4: m v$ ?

84 Oscar: Yeah, $m v$, right? Okay, now you 
85 have two of them, right? So what's the

86 momentum of each one of them? They're

87 identical bowling balls moving at the

88 same velocity in the same direction.

89 S1: Two $m v$ ?

90 Oscar: Okay, so two, so is that, so is that

91 for one of them now, or both of them

92 together?

$93 \mathrm{~S} 1$ : Both of them together.

94 Oscar: Together, right. Right, so I mean,

95 if you have each one of them going,

96 doing the exact same thing, same mass,

97 and you step far away, back enough-

98 S1: Mm-hmm.

99 Oscar: You know, you look at it, you

100 can't tell them apart, right?

101 S1: Yeah.

102 Oscar: But you can say, well, you know,

103 it's just got a total mass of ...[3 s

104 pause] What's the total mass?

$105 \mathrm{~S} 1$ : Of two $m$.

106 Oscar: Two $m$, right? But your velocity's

107 still the same?

108 S1: Yeah.

109 Oscar: Or different? Yeah. So same,

110 same velocity, twice the mass, so that's

111 the total momentum.

112 S1: Got you.

113 Oscar: So the question is, then, so you

114 can break it up that way for mass. How

115 can you break it up for velocity?

116 S1: I don't know.

117 Oscar: Any ideas? [13 s pause] I

118 can't. I can't think of any, well I can, but

119 it's a really weird thing. If I use, it's, um,

120 if you have, if you have advanced

121 calculus, there's a way to do it. But I

122 mean it doesn't really make much sense.

123 Right?

124 S1: Right.
125 Oscar: It's like, you know, I mean mass

126 you can imagine, okay, so I have one pen

127 and two pens. Those are two different

128 masses, right?

129 S1: Yeah.

130 Oscar: Right, I can put them together and

131 they got a total mass, but you can't really

132 do that with velocity.

a. Links physics and everyday experiences in a different way than the tutorial developers. The length of this episode is necessary to understand what Oscar is doing because the purpose of his questioning is apparent only at the end of the episode. At the start of the interaction, Oscar is asking questions about the momentum equation. These questions elicit the students' reasoning about what momentum depends on (mass and velocity) and why the students would expect those quantities to be multiplied rather than divided or subtracted (lines 1-45). This is aligned with the intentions of the tutorial, which aims to help students identify their intuitions about momentum and then relate them to physics. However, after this series of questions (and a short diversion into the definition of a vector), Oscar spends a significant portion of his time (a full half of the four minutes he is at the table) discussing an example that he introduces. By the end of the example, we can see that the purpose of it is to show the students why momentum should depend linearly on mass and velocity. This relationship is not emphasized in the tutorial and there is no indication that the students are particularly concerned about it, but the amount of time he devotes to discussing it shows its importance to Oscar.

Oscar's focus on the linear relationship of mass and velocity in the momentum equation demonstrates the difference in how he and the tutorial developers view the role of common sense in physics. As discussed in the previous example, Oscar does not consider common-sense ideas to be a sufficient foundation for building physics knowledge. While this tutorial seeks to begin with common-sense ideas of momentum and then relate them to the equation, Oscar wants students to begin with making sense of the equations and then to check that they are consistent with everyday life. There are many ways Oscar indicates that common sense is not the correct starting point. He asks the students about "momentum" and does not use the word oomph (line 6). He prompts them for the equation rather than a conceptual explanation: when the students say that momentum is $p$, Oscar asks what $p$ equals and not what $p$ is (line 8). He discusses examples but includes an example, dividing momentum into two separate velocities (lines 113-122), which is nonsensical. These actions allow Oscar to show the students that the equation can be connected to their everyday experiences but only after they have mathematically understood the formula for momentum.

$b$. Provides the assistance he thinks students need to construct their own knowledge. Because Oscar believes that tu- 
torials are too difficult for his students (as he made clear in his interviews), his role in his conversations with students is to provide some of the scaffolding steps for them to complete their assignment. In both of the examples of Oscar teaching, the conversation is directed by him; he asks the questions and the students provide the answers. He introduces the situations he would like to discuss and questions the students to highlight the points he thinks are important. In the previous example this includes the idea that momentum is a vector and that it is linearly dependent on velocity and mass. By directing the flow of conversation, Oscar can "take them by the hand and lead them through the steps at first," a course of action he thinks is necessary to counteract the tutorials' overestimation of the students' abilities.

In his interviews Oscar also discussed the role of the TA as providing necessary information for the students. He values group work because students can help answer each other's questions, which helps both the questioner and answerer learn. But he also thinks that when the entire group encounters a question that they cannot answer it is the job of the TA to help them through it. His view that the TA's role is to help solve problems too challenging for the group may explain why he asks questions with specific answers rather than asking open-ended questions.

c. Uses questions to guide students. We also see Oscar acting in accordance with the aspects of tutorials that he does value. In both of the episodes above, as in most of his interactions with his students that we have examined, Oscar questions his students rather than delivering "minilectures." This is consistent with his idea that students need to build their own knowledge and that questions are one of the ways TAs can assist students. While the same information can be imparted to students through a minilecture or the style of leading questions that Oscar uses, Oscar's choice of guided questions reflects his belief that if you tell students, they only receive the information once, but if they figure it out themselves, they can recreate that knowledge whenever they need it. Oscar is attempting to scaffold his students' construction of physics knowledge, but because he has specific goals of what he wants them to learn, the conversations he leads are quite rigid.

The previous section's examination of Oscar in the classroom shows the way his buy-in affects his classroom practice. His belief that students should construct their own knowledge leads to his frequent use of questions in student conversations. The fact that he expects particular answers to these questions can be connected to his idea that a TA needs to provide concrete help when a group is stuck and to his view that tutorials are too difficult for students. His focus on building physics meaning from equations rather than everyday experiences causes him to modify the focus of a tutorial. Oscar's specific beliefs about reform instruction can be connected in a fine-grained way to his instructional moves in a way that could not be captured, say, by a survey or observation protocol that classifies instructors along a constructivist or transmissionist spectrum.

\section{The interaction of buy-in and teaching practice}

In the two episodes presented here, Oscar's beliefs affect his actions. However, as discussed at length in other work, ${ }^{44}$ we are not telling a causally unidirectional story of beliefs driving behavior. Oscar's framing of his interaction with his students also gets stabilized by feedback loops that form, over both short and long time scales, between his beliefs, his focus of attention, and his actions. The Newton's third law tutorial, discussed above, provides an example. Believing that refining everyday thinking is unproductive, Oscar tells students not to take seriously the tutorial's call for commonsense reasoning. When he later interacts with a group of students, he glosses over rather than further eliciting the students' initial intuitive reasoning about less mass leading to a greater change in velocity (Sec. VI B 1 b, lines 1-8). Then he rejects the students' request to talk about inertia (lines 12-18), which also might have connected to the students' common-sense ideas about the effects of less mass or inertia. What he notices and amplifies instead are students' protestations that the questions "make us feel stupid" and their ability to figure out answers in response to his questions (e.g., lines 49-53). Oscar's beliefs "filter" his attention in a way that he does not fully "hear" or follow up on students' productive common-sense reasoning; instead, he hears student utterances supporting his view that leading students through his (as opposed to the tutorial's) way of approaching the topic is productive. In this way, a feedback loop begins to form between his initial beliefs about the inefficacy of building physics concepts from common-sense ideas, his lack of attention to common-sense aspects of students' reasoning, and his guided Socratic approach to questioning. Oscar's belief helps to cause the lack of attention (why attend to something that is unhelpful to students) and the lack of attention ensures that he does not hear student reasoning that would challenge his belief-i.e., students productively building on common sense. His belief also helps to drive his Socratic questioning, which then supports his belief; he sees students arrive at correct answers to his lines of questioning and does not see-because his Socratic questions do not give students the opportunity to express-productive common-sense reasoning that could be built upon. These bidirectional causal links between Oscar's beliefs, attention focus, and actions lead to a stable local coherence in his epistemological (and social) framing of his activity. The formation of this stable frame is part of the mechanism by which Oscar's lack of buy-in (as encoded in certain beliefs) leads to instruction contrary to the developers' intentions.

\section{COMPARISON OF TA BUY-IN ACROSS TWO INSTITUTIONS}

The above example shows how a TA may communicate his perceptions of the tutorials' value to students in his classroom, thus supporting or undermining the tutorial process. We have found that at the University of Maryland, most TAs do not buy into at least a few aspects of tutorial instruction, and this lack of buy-in aligns with their behavior in tutorial. As part of our research we wanted to better understand what contributes to TAs buying into tutorials, so as to better foster 
that in their professional development. For this purpose, we visited an institution at which most TAs seem to regard the tutorials as valuable and worthwhile (CU). We spent four weeks interviewing TAs and observing and videotaping the tutorial system there, including TA preparation sessions and multiple tutorial sessions.

To better characterize the TAs' buy-in, we examined the statements TAs made about tutorials in their interviews. We categorized those statements and produced a chart summarizing each TA's degree of buy-in to eight aspects of the tutorials discussed by the TAs during interviews. (These were aspects such as such as group work, conceptual emphasis, level of challenge, and so on.) Appendix A, which also discusses our coding methods, shows results for 15 UM TAs and 4 CU TAs.

A comparison of TAs' responses at the two institutions shows distinct differences. One-third of the UM TAs did not buy into one half or more of the attributes of tutorials. The CU TAs made comments that indicated their buy-in or mixed feelings about the majority of the tutorial attributes. A significant portion of UM TAs did not buy into two aspects that tutorial developers consider particularly important: the focus on qualitative reasoning and the importance of intuition in building physics knowledge. In contrast, the only category that CU TAs were predominantly not bought into reflects their concerns that tutorial questions are not always clearly worded, a concern that does not seem as critical to the successful implementation of tutorials as support for the content is. These findings support our initial observations that there is more buy-in at CU than at UM.

\section{EFFECT OF TUTORIAL SOCIAL AND ENVIRONMENTAL CONTEXT ON TA PERCEPTIONS OF THE TUTORIALS' VALUE}

A small number of studies examine the effects of social and environmental context on individual instructors. While none focus on TAs, research examining the effects of social and environmental context (variously termed the teaching environment, department-level culture, or situational characteristics) on professors and teachers has produced findings consistent with those presented in this paper. A large-scale survey of Australian professors found evidence that departmental policies and values affected chosen teaching approaches, such as the degree of focus on students. ${ }^{45}$ Professors have also identified these influences on their teaching approaches and priorities in interviews. ${ }^{46,47}$ A more detailed look at contextual effects on individual teaching practices is found by Henderson and Dancy, ${ }^{48}$ who found that teachers' conceptions of teaching were more aligned with reform instruction than their teaching practices (a finding supported by a multitude of K-12 teacher studies); ${ }^{49-54}$ the instructors were often aware of this inconsistency, which they explained by citing constraints of the context. These studies all assessed the instructors' perceptions of the context, in contrast to this study, in which descriptions of the social and environmental context are generated by the researchers.
Section VIII details the differences in tutorial social and environmental context that we have noted during our studies of CU and UM, which we feel can plausibly explain the differences in TA buy-in at the two institutions. The tutorial programs at UM and CU are in many ways very similar. As described in previous publications, ${ }^{1,2,55}$ students attend a one hour weekly tutorial in place of the discussion section as a component of the introductory year of physics courses. The TA professional development programs at these institutions are not described in detail in published literature ${ }^{1,38}$ but are also similar, bearing a strong resemblance at least superficially to the program at University of Washington (UW) on which both are based. ${ }^{56}$ Both programs employ physics graduate students mainly in their first and second years of studies and rely primarily on students who are not affiliated with their respective PER groups. The backbone of the professional development program is the weekly tutorial preparation sessions that are required for all TAs. During these sessions, TAs work though each week's tutorial themselves, learning the physics as well as the issues students commonly face with the material. Experienced TAs and faculty model effective instructional practices.

We have shown that, in spite of the apparent similarities in their situations, TAs at $\mathrm{CU}$ buy into the tutorials more highly than those at UM. In what follows, we describe the aspects of the two systems that seem to affect the experience of being a tutorial TA at each institution. In this section, unlike previous sections, we are not documenting specific causal connections. Instead we are noting the differences in institutional environments and making plausible arguments about their effects on TAs. We follow Pollock and Finkelstein ${ }^{2}$ in considering five levels of implementation of the tutorial program: levels of task formation, the classroom situation, the course culture, the department, and the university. In each case we observe the differences between UM and $\mathrm{CU}$ implementations that seem to affect the value that TAs place on tutorials. These differences are summarized in Table I.

\section{A. Task formation \\ 1. Production value}

Because CU uses the professionally published Tutorials in Introductory Physics ${ }^{5}$ and UM uses locally developed tutorials, there are a number of differences between these curricula that would be apparent even to TAs unfamiliar with the development of each set of tutorials. Most obviously, one is professionally published and one is inexpensively bound by the local copy center. We speculate that these features may contribute to TA buy-in at $\mathrm{CU}$ because the tutorials there appear to be (as they are in reality) a research-based curriculum developed by another institution and distributed to an extensive number of institutions, while the UM tutorials may be perceived as a pet project of the local PER group.

\section{Level of difficulty}

The content of the tutorials is also different in the two cases. The tutorials used at $\mathrm{CU}$ are calculus based and are 
TABLE I. A summary of the differences in social and environmental context at UM and CU and their likely effects on TAs.

\begin{tabular}{|c|c|}
\hline $\begin{array}{l}\text { Level of } \\
\text { implementation }\end{array}$ & University of Maryland \\
\hline \multirow[t]{5}{*}{ Task } & Locally produced \\
\hline & Algebra based \\
\hline & Often finished within allotted tin \\
\hline & $\begin{array}{l}\text { Uses informal terms such as } \\
\text { "oomph" to connect physics to } \\
\text { everyday understanding }\end{array}$ \\
\hline & Explicit epistemological focus \\
\hline \multirow[t]{2}{*}{ Situation } & 24 students in a room \\
\hline & $\begin{array}{l}\text { Off an isolated little-used } \\
\text { hallway }\end{array}$ \\
\hline \multirow[t]{5}{*}{ Course culture } & $\begin{array}{l}\text { Tutorial attendance only } \\
\text { recommended, not required }\end{array}$ \\
\hline & No credit given for tutorials \\
\hline & $\begin{array}{l}\text { Exam questions on tutorials } \\
\text { rarely used }\end{array}$ \\
\hline & $\begin{array}{l}\text { Students are primarily premed } \\
\text { and biological science } \\
\text { majors }\end{array}$ \\
\hline & $\begin{array}{l}\text { TAs in prep meetings } \\
\text { participate minimally }\end{array}$ \\
\hline \multirow[t]{4}{*}{ Department } & $\begin{array}{l}\text { Tutorial TAs also teach and } \\
\text { grade laboratories and } \\
\text { two kinds of homework }\end{array}$ \\
\hline & $\begin{array}{l}\text { Non-PER faculty may ignore } \\
\text { (or disparage) tutorials }\end{array}$ \\
\hline & $\begin{array}{l}\text { Tutorial teaching is } \\
\text { informally considered a } \\
\text { heavier teaching load }\end{array}$ \\
\hline & $\begin{array}{l}\text { A significant number of the } \\
\text { TAs are PER graduate } \\
\text { students }\end{array}$ \\
\hline
\end{tabular}

Likely effects on TAs

Professionally bound

Calculus based

Not finished within allotted time

Rigorous use of vocabulary and concepts

No explicit focus on epistemology

Room divided into bays where multiple tutorials occur at once

Large, bright, noisy room

Tutorial attendance required

Small amount of grade based on participation

Tutorial material is $25 \%$ of the exam

Students are primarily engineering and natural science majors

TAs may buy in more to tutorials they perceive as challenging, rigorous, and undiluted
CU tutorials are a highly communal experience; UM tutorial conveys a feeling of isolation

TAs may buy into tutorials more when they directly affect students' grades and link to other parts of the class. Their participation in the prep meetings is consistent with their varying buy-in

TAs in prep meetings demonstrate more authentic participation

Tutorial TAs only teach tutorials and grade only tutorial homework

Lecturer and tutorial instructor are non-PER

Lecturer appears frequently at TA meetings
TAs may buy in more when they perceive the tutorials to be part of the accepted departmental practice

University

LA program trains undergraduates who assist TAs in tutorials
Institutional support can also be communicated through universitywide programs typically longer. They are more difficult for students to complete and are in fact designed so that no student group will finish and be unoccupied during part of the period. In contrast, UM tutorials were written for use in an algebra-based course and it is not unusual for some student groups to complete the entire tutorial during the session.

\section{Purity of physics content}

The CU tutorials are more exclusively physics oriented than UM tutorials, whose epistemological emphasis allows for inclusion of questions that encourage students to reflect on their learning processes as well as on the physics concepts. The explicit epistemological component of the UM 


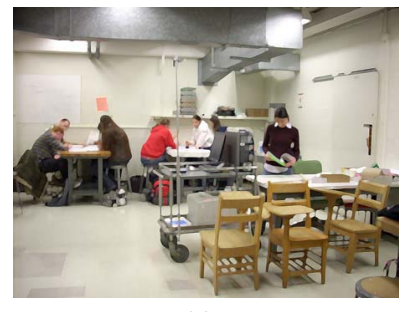

(a)

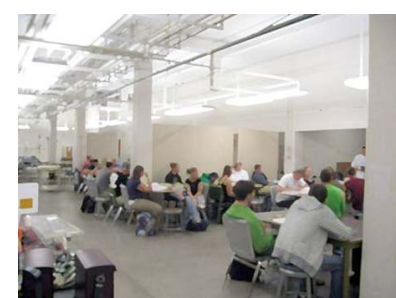

(b)
FIG. 2. (Color) The photo on the left shows the UM tutorial room, which is located off a little-used hallway. The photo on the right shows the CU tutorials, which are conducted in three adjacent bays located in a cavernous room in which many other classes are taught.

tutorials is salient for the TAs using them. In interviews where TAs discuss their tutorial experiences, one UM TA talked about a tutorial activity in which students are asked to consider their own ideas about learning physics: "I meanwell, physics is really a very precise science, right? So I mean people's opinion doesn't matter that much. So I mean they should-I mean it's better if they have the impression that there is actually something that's absolutely right in physics." Comments like this show that the epistemological component of the UM tutorials can be perceived as too easy, a poor use of time, or even harmful for students. In comparison, CU TAs do not remark on the exclusive focus on physics in the tutorials they use. TAs' lack of familiarity with explicit epistemological instruction may cause them to value it less, leading to less TA buy-in at UM.

All of these features contribute to TAs valuing the tutorials used at CU more highly than those used at UM. The tutorials used at CU are professionally produced, more challenging, and not diluted by questions that are not specifically about physics. These tutorials are also more formal in the sense that they do not introduce nonphysics terms such as oomph and they do not end a section before a formal concept has been developed.

\section{B. Level of situations}

\section{Classroom location and appearance}

The classroom contexts for the tutorials at the two institutions are also different. At UM, all tutorial sessions (about 20 in the course of a week) are all held in the same classroom, a windowless minimally maintained laboratory room off a little-used hallway with a capacity of about 24 students. At CU, tutorials are held in a large room divided into bays; each tutorial session takes place among other simultaneous tutorial sessions in a crowded, open, noisy setting connected by a well-traveled corridor. The CU setting potentially displays the tutorials as being a highly central and communal experience, something that many others are actively engaged in at the same time. The UM setting, in contrast, is isolated from other physics instruction happening in the building. Figure 2 shows the two rooms.

\section{Course culture}

\section{Attendance requirement}

The TAs' perceptions of the tutorials are also influenced by how the courses are structured at each institution. Partici- pation in the tutorials is required at $\mathrm{CU}$; a small percent of the students' grade is based on this participation. At UM, tutorial attendance is recommended, but no credit is given and the amount of encouragement to participate varies among lecturers.

\section{Representation on exams}

The emphasis on the conceptual reasoning practiced in tutorials is also different. At UM, exam questions based on tutorial material are available to all lecturers but are mainly used by those faculty affiliated with the PER group. At CU, questions on tutorial material generally comprise $25 \%$ of each exam grade and both TAs and students are aware of this. Together, the lack of attendance credit and dedicated exam questions lead UM TAs to discount the importance of tutorials. This difference in perception was reflected in the way TAs evaluated the tutorials' "fit" with the rest of the course components. At UM, three-quarters of the TAs described the tutorials as disconnected from the rest of the course or as not preparing students for their exams or homework. In contrast, only one CU TA raised the issue, and he believed that the tutorials provided preparation for a sizable component of the course assessments.

\section{Student population}

The student population of each class varies as well: at $\mathrm{CU}$, the tutorials are used in the calculus-based introductory courses for engineering and natural science majors while the UM class is algebra based and taken by premeds and biological science majors. This difference may result in TAs more highly valuing instruction that is more math intensive and is offered to physics students.

\section{TA preparation meetings}

At both institutions TAs attend weekly meetings to prepare for the following week's tutorial. The CU tutorial supervisor was a researcher not associated with the PER group. The UM tutorial supervisor was a PER researcher one year and an unaffiliated postdoctoral researcher the second year. A cursory overview of the meetings at CU and UM would not reveal any startling differences. In each, the tutorial supervisor introduces the tutorial and sometimes leads a discussion. Then the TAs work in small groups, as their students will, to answer the questions on the worksheet while the tutorial supervisor circulates, modeling the instructional practices he or she would like the TAs to use.

A more detailed examination shows important differences. To illustrate this, a video clip of a TA group in each university's preparation session was selected and the clips were compared. While we attempted to choose video clips that seemed representative, the clips were not chosen randomly and the selection could have been influenced by researcher preconceptions. In the UM clip, three TAs answer the questions on the worksheet and do some of the experiments described in the tutorial. While they offer comments and questions, the conversational turns are short and are rarely in response to other comments and they make little eye contact, so that there is minimal continuity in the conversation. None 
of the TAs writes anything on their worksheets and they do not work on the questions simultaneously. The objective of the TAs appears to be to become familiar with the exercises and equipment and it seems that perfunctory participation is sufficient to achieve this objective. In contrast, the CU clip shows two TAs and two LAs answering the same questions at the same time. They discuss the question they are working on and respond to each other's questions and comments. There is more continuity in their conversation and they are attempting to answer all the questions on the tutorial. The tutorial appears to be taken seriously as a way that they can better understand physics and as a challenging experience for their students for which they need to prepare. We conjecture that because UM TAs buy into the tutorials less thoroughly, their attitude is one of the factors that make the meetings worse; the decreased quality of the meetings then negatively impacts other TAs in a feedback loop. The striking observation, though, is the distinctly different TA behavior that occurs in spite of the similar structure of the two meetings. Because the meeting agendas are so similar, it seems likely that social and environmental context aspects beyond the preparation sessions affect the TAs' actions.

\section{Department level}

\section{Nature of TA assignment}

At the departmental level, a distinct difference between the two tutorial implementations is that CU TAs assigned to teach the introductory course teach multiple tutorial sections, but no laboratory sections. They are responsible for grading only tutorial homework and class exams. At UM, a TA with a full TA assignment typically teaches two tutorial sections and four hours of laboratory and grades laboratory reports, tutorial homework, quantitative homework, and class exams. As a result, the tutorial instruction is only a fraction of a UM TA's responsibilities. It is possible that this contributes to lower UM TA buy-in, and at a minimum it requires them to divide their attention. In addition, because first-year UM TAs attend a mandatory department-wide professional development meeting, a first-year tutorial TA at UM attends three and a half hours of weekly preparatory meetings, while a CU TA attends one and a quarter hours weekly. The large amount of mandatory meeting time for TAs of this course leads to it being informally considered a heavier teaching load than the average TA assignment, and it is possible that this also contributes to lack of TA buy-in.

\section{Support by regular faculty}

The CU implementation has achieved a higher level of independence from the PER group that advocated their introduction as compared to the UM execution. At $\mathrm{CU}$, the lecturer of the course associated with tutorials is not a member of the physics education group, but is informed about and supports tutorials. During the month that we observed, he often appeared at the TA preparation sessions. The tutorial supervisor was a researcher, not associated with the PER group, who ran the preparation sessions competently. At UM, there are typically three lecturers teaching the introductory course that uses tutorials. The majority of these lecturers are non-PER and they consider tutorial preparation to be solely the responsibility of the tutorial supervisor. In addition, the position of tutorial supervisor, which in the earlier years was filled by a member of the PER group, was assigned during the second year we collected data to a postdoctoral researcher outside of PER who had no previous experience with tutorials.

\section{PER group involvement}

Another factor that may communicate the department's support of tutorials to the TAs is the involvement of PER graduate students as tutorial TAs. At UM, PER graduate students often volunteer to teach tutorials. At $\mathrm{CU}$, one or two PER graduate students who do not have research positions yet may be assigned to teach tutorials, as any unfunded graduate student would. The fact that one-third of the tutorial TAs at UM can be affiliated with PER may contribute to the UM TAs perception that tutorials are a PER-supported project rather than one supported by the whole department, as at CU. ${ }^{57}$

\section{E. University level}

\section{Interdepartmental reform effort}

Support for reform instruction is also present at the university level at CU. Their Learning Assistant (LA) program $^{2,55,58}$ selects students who are high achievers in the introductory classes to assist TAs in teaching tutorials. The physics LAs also take a course with LAs from other STEM disciplines, in which they reflect on their teaching and study teaching methods and theories of learning. The existence of this program, which provides an LA to teach with each TA in the CU tutorials, is one of the elements that may communicate to the TA the value that the university places on reform instruction.

\section{CONCLUSION}

Physics graduate students' beliefs about how physics should be taught affects their teaching. For example, Oscar's belief that knowledge construction should begin with equations leads him to disregard students' common-sense ideas and his belief that TAs should provide concrete help leads to his guided Socratic questioning. The example of Oscar's teaching suggests that buy-in is a necessary (but insufficient) component of effective curriculum implementation.

The weekly curriculum-based professional development programs commonly offered to graduate student TAs appear to have limited impact on the TAs' buy-in of the curriculum in use. These meetings, using a typical combination of pretests, Force Concept Inventory data, and working through tutorials, can help familiarize TAs with the content they will be teaching and with some typical difficulties students encounter when learning that material. While such preparation is necessary for effective teaching, it is not enough. The commitment to teach in a reformed manner and the skills needed to do that are also important. Our analysis suggests that these programs cannot instill the necessary valuation of 
reform teaching. A goal of our future research is to determine what sorts of activities would be most effective in scaffolding such values.

Effective professional development for TAs can be informed by detailed understanding of TA beliefs and motivations. Oscar, for example, believes that students should construct their own knowledge; a professional development program well suited to Oscar would build on that belief. It would also address Oscar's concern that tutorials are too difficult for students. Broad characterizations of TAs as "not buying in" risk obscuring valuable information about specific attitudes and skills that TAs already have.

The tutorial social and environmental context, including the classroom, departmental, and institutional environments, affects the beliefs that TAs at a particular institution hold. Greater attention to the development of supportive social and environmental context can help tutorial TAs value the tutorials they are asked to teach. The nature of this attention is likely to be specific to local circumstances. The analysis presented here suggests that TAs absorb the implicit attitudes of their colleagues and department. If TA supervisors ignore these implicit messages, TAs will be less likely to engage in effective reform teaching.

\section{ACKNOWLEDGMENTS}

We are grateful for input from the Physics Education Research Groups at the University of Colorado, Boulder and the University of Maryland, College Park. We especially thank Chandra Turpen for valuable feedback on this paper. This material is based on work supported by the National Science Foundation under Grants No. REC 0529482 and No. 0715567.

\section{APPENDIX A: CHARACTERIZATION OF TA BUY-IN}

In order to better understand the individual TAs' buy-in, their transcribed interviews were coded. The interview questions were open ended in order to respond to TAs' replies. A typical question was "What do you see as the advantages and disadvantages of tutorial-style teaching, for you, and for the students?"

To develop categories, we examined a subset of TA interviews, selected quotes in which they were discussing aspects of tutorials, and then created categories from them. Thus, the categories are a reflection of the characteristics of tutorials that TAs considered noteworthy rather than the aspects of tutorials that the developers value. After these categories were established, we coded all the transcripts from TA interviews.

If a TA discussed some aspect of tutorial, that talking turn was categorized. Individual turns were put into multiple categories when appropriate. All interviews turns were sorted into one of the categories or coded as not relating to tutorials (an example of the latter would be a discussion of how the TA learns best). Each comment was labeled as predominantly showing buy-in (aligning with the developers' ideas), predominantly not showing buy-in (not aligning with the developers' ideas), or as mixed. All of the comment ratings in a category were considered together to determine a rating for each TA in each category. (Again, they were rated as predominantly showing buy-in, anti-buy-in, or as a mix.) If a TA did not mention that aspect, there is no code for that TA in that category.

One researcher did all of the coding. To check inter-rater reliability, a second researcher was given one hour of training and then was given 21 quotes to which the first researcher had assigned at least one category. For each quote, the second coder assigned categories to the quotes and coded whether the TA was bought in, not bought in, or "mixed" with respect to each category.

For the buy-in codes, there was $86 \%$ agreement, with no disagreements between "buy-in" and "anti-buy-in." Instead, all the disagreements were between mixed and one of the other two categories.

The coders agreed on the categories assigned $79 \%$ of the time, but about half of the mismatches were due to a disagreement about whether a second category needed to be assigned (e.g., the first coder assigned two categories while the second coder assigned just one). In those cases, the coder who assigned just one category was asked to assign a secondary category. The secondary category chosen agreed, in two of the three cases, with the secondary category assigned by the other coder. In summary, the two coders disagreed on categories $21 \%$ of the time: $12 \%$ were disagreements about category choices and 9\% were disagreements only about whether the "signal" from a secondary category was strong enough to warrant a category assignment.

Table II shows the designation each TA received in all of the categories on which he commented. The designations are indicated with colors: the lightest for buy-in, the medium shade for mixed (both aligned and nonaligned comments), and the darkest for anti-buy-in. A TA's comments are considered mixed if less than approximately three-quarters of the comments in that category were aligned (or not aligned). If a category had no comments from a TA, the corresponding box is hatched.

As an example, consider Chris, the UM TA shown in the fifth column, labeled "C." When he discussed group work, he said that he valued it for students because it gave more of them a chance to ask questions, allowed them to teach each other, and provided them with the chance to focus on their own particular difficulties. He also appreciated it as a teacher because he did not have to devise a "50 minute show" and because it better prepared him to answer the questions he would expect when he was a lecturer. His only concern was that having to answer student questions on the spot took more time than delivering a prepared lecture. Because his comments were predominantly aligned, his rating for this category was buy-in.

Chris was concerned that the qualitative focus of the tutorials did not prepare students sufficiently for the Medical College Admission Test and their quantitative multistep homework problems. He did not suggest any positive aspects of the emphasis on qualitative physics reasoning. As a result, he was rated as anti-buy-in for this category.

Chris's assessment of the structured nature of tutorials was mixed. Because the tutorials were a prepared curriculum, Chris liked the limited preparation required but found it 
TABLE II. (Color) Designation each TA received in all of the categories on which he commented.

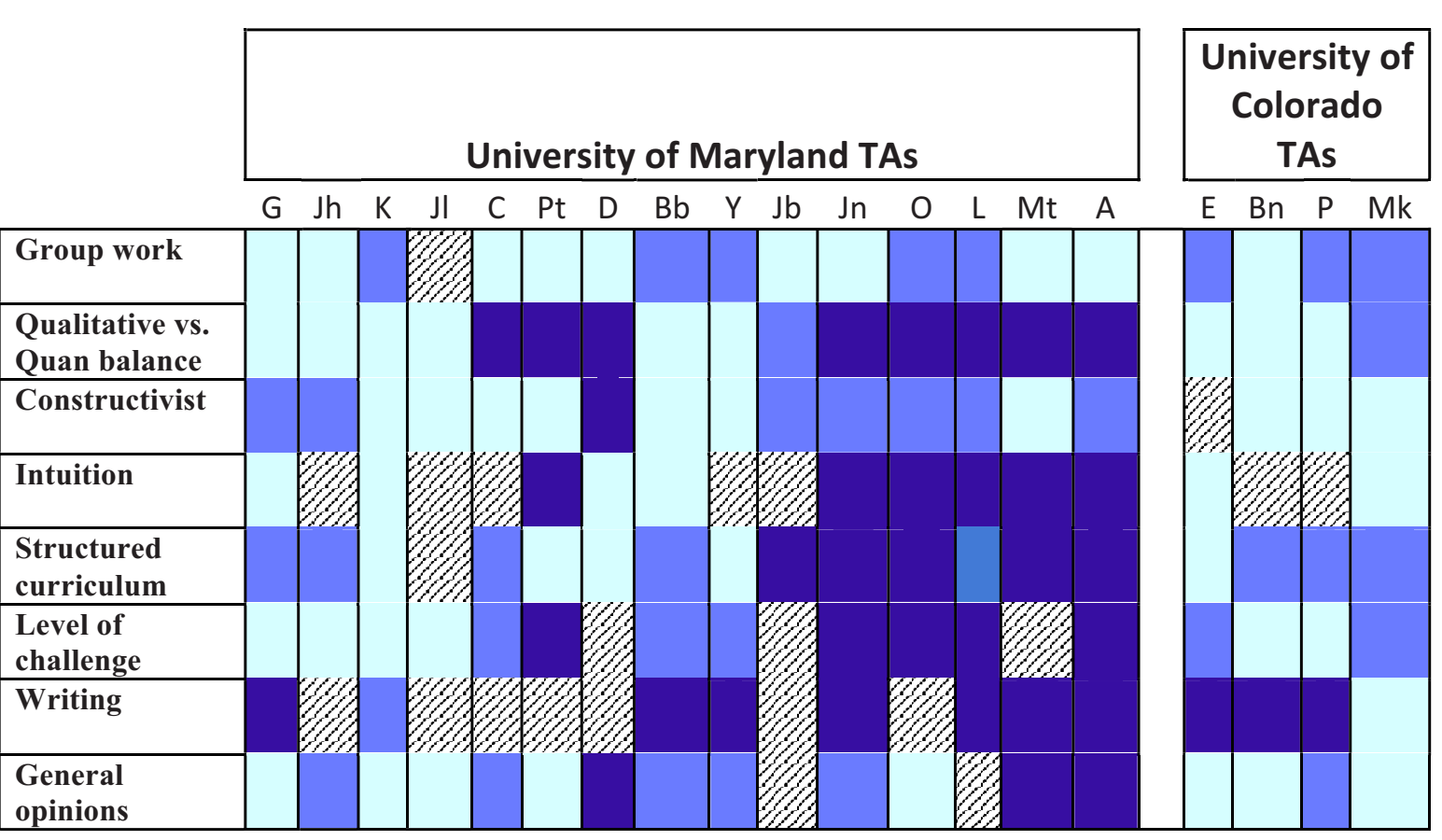

\begin{tabular}{|l|l|}
\hline & Buy-in \\
\hline & Mixed \\
\hline & Anti-buy-in \\
\hline $\mathscr{Z Z}$ No data for this category \\
\hline
\end{tabular}

difficult to use material that was unfamiliar. He said that at the beginning, it "was kind of hard to be using someone else's words effectively, and I kind of got a handle on that and also got a handle on how to put my thoughts in it...." His buy-in that category was accordingly rated as mixed.

The UM TAs were interviewed twice at the start and end of the semester they taught. The comments from these two interviews were combined before they were rated. The CU TAs were interviewed once near the end of the semester they taught. We might expect that a grouping of initial and final assessments would obscure changes that occurred in UM TAs during the semester they taught. In order to estimate how much change might have occurred, we counted the number of times that we could have observed a change (i.e., the number of times a TA commented on a particular category in both the initial and final interviews), which was 57 instances. We then tallied the number of times our codes of a TA's values changed, for example, from mixed to positive, which happened 17 times. This means that changes in TAs' values occurred about $30 \%$ of the time, where about twothirds of the observed changes were positive (i.e., from mixed to positive or negative to mixed). This is not an extensive amount of change, and it is consistent with our informal observations.

\section{APPENDIX B: INTERVIEW QUESTIONS}

The following questions were asked during the openended interviews we conducted with the TAs.

1. Have you taught before?

2. How is the course going?

3. How is discussion section going?

4. What do you see as the advantages and disadvantages of tutorial-style teaching: for you? for the students?

5. How would you recommend tweaking the current format?

6. Do you think your students are learning better, worse, or the same as they would in a regular-style recitation section?

7. What's hard about teaching this way, and what's easy?

8. Do you think that the course (lecture and tutorials) help teach what students should be learning in a physics course?

9. (Asked only in the 2007 and 2008 interviews) When teaching tutorials, what did you see your job as? 
${ }^{1}$ N. Finkelstein and S. Pollock, Replicating and understanding successful innovations: Implementing tutorials in introductory physics, Phys. Rev. ST Phys. Educ. Res. 1, 010101 (2005).

${ }^{2}$ S. Pollock and N. Finkelstein, Sustaining educational reforms in introductory physics, Phys. Rev. ST Phys. Educ. Res. 4, 010110 (2008)

${ }^{3}$ C. Crouch and E. Mazur, Peer instruction: Ten years of experience and results, Am. J. Phys. 69, 970 (2001).

${ }^{4} \mathrm{P}$. Heller and M. Hollabaugh, Teaching problem solving through cooperative grouping. Part 2: Designing problems and structuring groups, Am. J. Phys. 60, 637 (1992).

${ }^{5}$ L. McDermott, P. Shaffer, and P. E. G. U. o. Washington, Tutorials in Introductory Physics (Prentice-Hall, Upper Saddle River, NJ, 2002).

${ }^{6} \mathrm{D}$. Sokoloff and R. Thornton, Interactive Lecture Demonstrations, Active Learning in Introductory Physics (Wiley, New York, 2004).

${ }^{7}$ L. McDermott and P. Shaffer, Research as a guide for curriculum development: An example from introductory electricity. Part I: Investigation of student understanding, Am. J. Phys. 60, 994 (1992).

${ }^{8}$ P. Shaffer and L. McDermott, Research as a guide for curriculum development: An example from introductory electricity. Part II: Design of an instructional strategy, Am. J. Phys. 60, 1003 (1992).

${ }^{9}$ C. M. Ishikawa, G. R. Potter, J. A. Blickenstaff, C. J. De Leone, P. Castori, and W. H. Potter, Meeting of the National Association for Research in Science Teaching, New Orleans, LA, 2000 (unpublished).

${ }^{10}$ F. H. Lawrenz, P. Heller, R. Keith, and K. Heller, Training the teaching assistant, J. Coll. Sci. Teach. 22, 106 (1992).

${ }^{11}$ J. Luft, J. Kurdziel, G. Roehrig et al., Growing a garden without water: Graduate teaching assistants in introductory science laboratories at a doctoral/research university, J. Res. Sci. Teach. 41, 211 (2004).

${ }^{12}$ J. McGivney-Burelle, T. DeFranco, C. Vinsonhaler et al., Building bridges: Improving the Teaching Practices of TAs in the Mathematics Department, J. Grad. Teach. Assist. Dev. 8, 55 (2001).

${ }^{13}$ E. Price and N. Finkelstein, arXiv:physics/0609003 (unpublished).

${ }^{14}$ J. Belnap, Putting TAs into context: Understanding the graduate mathematics teaching assistant, Ph. D. dissertation, The University of Arizona, 2005.

${ }^{15}$ M. Volkmann and M. Zgagacz, Learning to teach physics through inquiry: The lived experience of a graduate teaching assistant, J. Res. Sci. Teach. 41, 584 (2004).

${ }^{16} \mathrm{~L}$. Cronin-Jones, Science teacher beliefs and their influence on curriculum implementation: Two case studies, J. Res. Sci. Teach. 28, 235 (1991).

${ }^{17}$ P. Peterson, Doing more in the same amount of time: Cathy Swift, Educational Evaluation and Policy Analysis 12, 261 (1990).

${ }^{18} \mathrm{~N}$. Wiemers, Transformation and accommodation: A case study of Joe Scott, Educational Evaluation and Policy Analysis 12, 281 (1990)

${ }^{19} \mathrm{~S}$. Wilson, A conflict of interests: The case of Mark Black, Educational Evaluation and Policy Analysis 12, 293 (1990).

${ }^{20}$ J. Haney, A. Lumpe, C. Czerniak, and V. Egan, From beliefs to actions: The beliefs and actions of teachers implementing change, Journal of Science Teacher Education 13, 171 (2002).
${ }^{21}$ V. Otero, Moving beyond the "get it or don't" conception of formative assessment,'J. Teach. Educ. 57, 247 (2006).

${ }^{22}$ N. Speer, Connecting Beliefs and Practices: A Fine-Grained Analysis of a College Mathematics Teacher's Collections of Beliefs and Their Relationship to His Instructional Practices, Cogn. Instruct. 26, 218 (2008).

${ }^{23}$ H. Borko and R. Putnam, Handbook of Educational Psychology, edited by D. Berliner and R. Calfee (MacMillan, New York, 1996), Vol. 2, p. 673.

${ }^{24}$ A. Lawson, R. Benford, I. Bloom et al., Evaluating College Science and Mathematics Instruction: A Reform Effort That Improves Teaching Skills, J. Coll. Sci. Teach. 31, 388 (2002).

${ }^{25}$ D. Sawada, M. Piburn, E. Judson et al., Measuring Reform Practices in Science and Mathematics Classrooms: The Reformed Teaching Observation Protocol, Sch. Sci. Math. 102, 245 (2002).

${ }^{26}$ T. Carpenter, E. Fennema, P. Peterson et al., Using knowledge of children's mathematics thinking in classroom teaching: An experimental study, Am. Educ. Res. J. 26, 499 (1989).

${ }^{27}$ C. M. Ezrailson, EMIT: Explicit modeling of interactiveengagement techniques for physics graduate teaching assistants and the impact on instruction and student performance in calculus-based physics, Ph. D. thesis, Texas A \& M University, 2004.

${ }^{28}$ C. Schroeder, T. Scott, H. Tolson, T.-Y. Huang, and Y.-H. Lee, A meta-analysis of national research: Effects of teaching strategies on student achievement in science in the United States, J. Res. Sci. Teach. 44, 1436 (2007).

${ }^{29}$ E. Close, School of Education, Doctor of Philosophy (Seattle Pacific University, Seattle, WA, 2008).

${ }^{30}$ Frames, scripts, and schemata are related and overlapping terms in the fields of linguistics, artificial intelligence, cognitive psychology, social psychology, sociology, anthropology, and other disciplines. An overview and history of the uses of these related terms appears in D. Tannen, Framing in Discourse (Oxford University Press, New York, 1993), Chap. 1.

${ }^{31} \mathrm{G}$. Bateson, Steps to an Ecology of Mind: Collected Essays in Anthropology, Psychiatry, Evolution, and Epistemology (Chandler Press, San Francisco, 1972).

${ }^{32}$ E. Goffman, Frame Analysis: An Essay on the Organization of Experience (Harper, New York, 1974).

${ }^{33} \mathrm{G}$. MacLachlan and I. Reid, Framing and Interpretation (Melbourne University Press, Portland, OR, 1994).

${ }^{34}$ D. Tannen, Framing in Discourse (Oxford University Press, New York, 1993).

${ }^{35} \mathrm{~N}$. Brickhouse, Teachers' beliefs about the nature of science and their relationship to classroom practice, J. Teach. Educ. 41, 53 (1990).

${ }^{36}$ D. Hammer, A. Elby, R. Scherr et al., in Transfer of Learning: Research and Perspectives, edited by J. Mestre (Information Age, Greenwich, CT, 2005), p. 89.

${ }^{37}$ F. Erickson, Talk and Social Theory (Polity Press Malden, Cambridge, MA, 2004).

${ }^{38}$ E. Redish and D. Hammer, Reinventing college physics for biologists: Expecting an epistemological curriculum, Am. J. Phys. 77, 629 (2009).

${ }^{39}$ L. McDermott, P. Shaffer, and M. Somers, Research as a guide for teaching introductory mechanics: An illustration in the context of the Atwood's machine, Am. J. Phys. 62, 46 (1994).

${ }^{40}$ A. Elby, Helping physics students learn how to learn, Am. J. 
Phys. 69, S54 (2001).

${ }^{41}$ A. Elby, R. Scherr, T. McCaskey et al., Maryland tutorials in physics sense making, DVD, funded by NSF DUE-0341447.

${ }^{42}$ J. Lave and E. Wenger, Situated Learning: Legitimate Peripheral Participation (Cambridge University Press, Cambridge, 1991).

${ }^{43}$ R. Scherr and A. Elby, in Physics Education Research Conference, edited by P. Heron, L. McCullough, and J. Marx (American Institute of Physics, New York, 2006), Vol. 883, p. 46.

${ }^{44}$ R. Goertzen, R. Scherr, and A. Elby Tutorial TAs in the classroom: Framing teaching as a focus on indicators(unpublished).

${ }^{45}$ P. Ramsden, M. Prosser, K. Trigwell, and E. Martin, University teachers' experiences of academic leadership and their approaches to teaching, Learn. Instr. 17, 140 (2007).

${ }^{46}$ P. T. Knight and P. R. Trowler, Department-level cultures and the improvement of learning and teaching, Studies in Higher Education 25, 69 (2000).

${ }^{47} \mathrm{M}$. Prosser and K. Trigwell, Relations between perceptions of the teaching environment and approaches to teaching, Br. J. Educ. Psychol. 67, 25 (1997).

${ }^{48}$ C. Henderson and M. Dancy, Barriers to the use of researchbased instructional strategies: The influence of both individual and situational characteristics, Phys. Rev. ST Phys. Educ. Res. 3, 020102 (2007).

${ }^{49}$ L. Bryan, Nestedness of beliefs: Examining a prospective elementary teacher's belief system about science teaching and learning, J. Res. Sci. Teach. 40, 835 (2003).
${ }^{50}$ D. K. Cohen, A revolution in one classroom: The case of Mrs. Oublier, Educational Evaluation and Policy Analysis 12, 311 (1990).

${ }^{51}$ M. G. Jones and G. Carter, in Handbook of Research on Science Education, edited by S. K. Abell and L. M. Lederman (Lawrence Erlbaum, Mahwah, NJ, 2007), p. 1067.

${ }^{52}$ K. King, L. Shumow, and S. Lietz, Science education in an urban elementary school: Case studies of teacher beliefs and classroom practices, Sci. Educ. 85, 89 (2001).

${ }^{53}$ P. Simmons, A. Emory, T. Carter et al., Beginning teachers: Beliefs and classroom actions, J. Res. Sci. Teach. 36, 930 (1999).

${ }^{54} \mathrm{~K}$. Tobin and C. McRobbie, Beliefs about the nature of science and the enacted science curriculum, Sci. Educ. 6, 355 (1997).

${ }^{55}$ N. Finkelstein, V. Otero, and S. Pollock, in APS Forum on Education, Fall 2006-Spring 2007, p. 11.

${ }^{56}$ The tutorial preparation in the first year we collected data was conducted by one of the authors, Rachel Scherr, who has extensive experience conducting TA tutorial preparation programs at UW.

${ }^{57}$ The involvement of numerous PER graduate students in tutorials does not necessarily lead to a lack of TA buy-in. At the University of Washington, all PER graduate students teach tutorials throughout their graduate careers in an implementation of tutorials that has lasted almost two decades.

${ }^{58}$ V. Otero, N. Finkelstein, R. McCray et al., Who is responsible for preparing science teachers?, Sci. Educ. 313, 445 (2006). 